used in Fig. 8. The Electric Power Research Institute provided support for analysis of experimental data related to liquefaction of coal.

\section{Nomenclature}

$G_{L} \quad=$ dimensionless cake thickness

$G_{t} \quad=$ dimensionless filtration time

$G_{v} \quad=$ dimensionless filtrate volume

$G_{t \mathrm{dis}}=$ dimensionless displacement time

$L \quad=$ cake thickness

$p \quad=$ applied pressure

$p_{m} \quad=$ pressure drop across medium

$R_{m} \quad=$ medium resistance

$t \quad=$ filtration time

$t_{\mathrm{dis}} \quad=$ displacement time

$v \quad=$ filtrate volume per unit area

$v_{c} \quad=$ cake volume per unit area

$v_{s} \quad=$ slurry volume in tank per unit area

$v_{F} \quad=$ feed slurry volume per unit area

$w \quad=$ mass of dry solids per unit area

$\alpha_{\mathrm{av}} \quad=$ average specific cake resistance $\varepsilon_{\text {sav }}$

$\eta$

$\mu$

$\rho_{s}$

$\sigma$

$\phi_{s}$

$\phi_{s F}$ $\chi$
$=$ average volume fraction of solids in cake

$=$ ratio of volume fraction of solids in cake to feed

$=$ viscosity of liquid

$=$ density of solids

$=$ specific gravity of solids

$=$ volume fraction of solids in tank slurry

$=$ volume fraction of solids in feed slurry

$=$ dimensionless medium resistance
$[-]$

$[\mathrm{Pa} \cdot \mathrm{s}]$

$\left[\mathrm{kg} / \mathrm{m}^{3}\right]$

[-]

$[-]$

[-]

$[-]$

Literature Cited

1) Jahreis, C. A.: Chem. Eng. Prog., 59 (5), 91 (1963).

2) Lewis, H. W.: "Solvent Refined Coal (SRC) Process, Operation of Solvent Refined Coal Pilot Plant at Wilsonville, Ala.", Quarterly Technical Report for the period April-June, 1976, Catalytic, Inc., Wilsonville, Ala. 35186

3) Tiller, F. M. (Ed.): “Theory and Practice of Solid-Liquid Separation"', 2nd Ed, p. 250, Dept. of Chem. Eng., University of Houston, Houston, 1975.

4) Toro, L. B.: MS Thesis, University of Houston, Texas (1965).

\title{
REMOVAL OF EMULSIFIED OIL PARTICLES BY DISPERSED AIR FLOTATION
}

\author{
YuJi SATO, Yasuhiro MURAKAMI, Tsutomu HIROSE, \\ YoshIFUMI URYU AND KoJI HIRATA \\ Department of Chemical Engineering, \\ Kyushu University, Fukuoka 812
}

\begin{abstract}
Dilute emulsified particles $\left(C \simeq 300 \mathrm{~g} \cdot \mathrm{m}^{-3}, D_{P} \simeq 8 \mu \mathrm{m}\right)$ of heavy oil A were removed in a 65 mm-diameter, $0.32 \mathrm{~m}$-deep column with a solution of $\mathrm{pH} 4$ which minimized double-layer repulsion.

Firstly, flotation by bubbling through glass frits of three different pore sizes shows that the removal rate is proportional to the oil concentration to the first order at low gas velocity $(0.01 \leqq$ $\left.U_{g} \leqq 0.05 \mathrm{~cm} \cdot \mathrm{s}^{-1}\right)$ and to the second order at high gas velocity $\left(0.1 \leqq U_{g} \leqq 3 \mathrm{~cm} \cdot \mathrm{s}^{-1}\right)$. This fact implies that the predominant rate step is oil particle-bubble collision in the former region and particle-particle collision in the latter. Mechanical agitation by a paddle gives the same removal rate as bubbling in the high gas velocity region when the power consumption per unit volume is the same. This fact implies that the role of bubbling there is to give adequate turbulence to the liquid.

Secondly, the bubble diameter was changed from $40 \mu \mathrm{m}$ to $2 \mathrm{~mm}$ under the mechanism of bubbleparticle collision by electrolysis, dissolved and bubbling air flotation. With increasing bubble diameter, the collection efficiency decreases rapidly $(\simeq$ to the -2 power $)$ for small bubbles and much more gradually for large bubbles. This trend coincides with the existing and proposed trajectory theories.
\end{abstract}

\section{Introduction}

Air flotation has been applied to the separation of emulsified oil from water. The operation may be

\footnotetext{
Received October 4, 1979. Correspondence concerning this article should be addressed to T. Hirose. Y. Sato is now with Kuken Kogyo Ltd., Fukuoka 810, Y. Uryu is now with Idemitsu Petrochm. Co., Ltd. Kimitsugun 192-01 and $\mathrm{K}$. Hirata is now with Sumitomo Bakelite Co., Ltd., Tokyo 131.
}

classified into (1) dissolved air flotation and (2) dispersed air flotation with respect to the generation of fine bubbles. In the former, the dissolving pressure is a decisive operating variable. Its effect was discussed in the previous paper ${ }^{6)}$, along with the effect of the liquid properties. In the latter, the gas flow rate may be an important operating variable, and the 


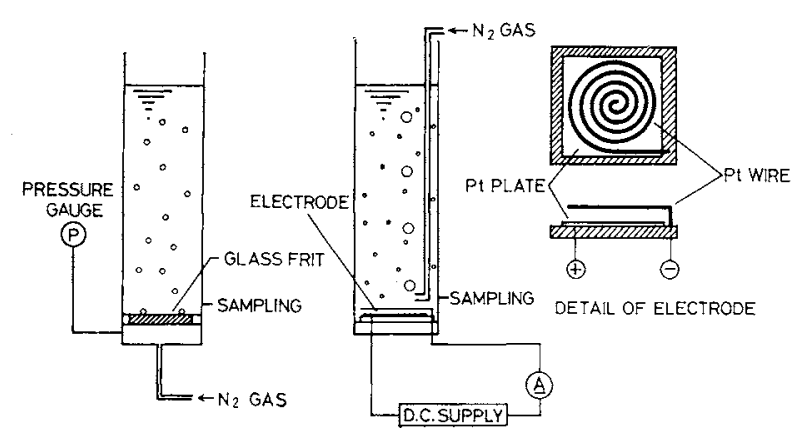

(a) Bubbling air flotation

(b) Electrolysis air flotation

Fig. 1 Schematic diagram of apparatus

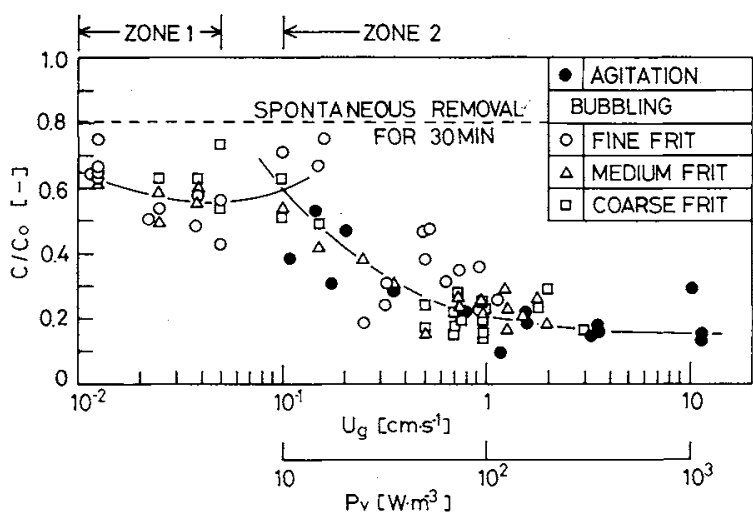

Fig. 2 Variation of residual oil concentration after 30 minutes' bubbling with superficial gas velocity in bubbling flotation

present investigation deals primarily with its effect on the flotation efficiency and the flotation mechanism.

Bubble size varies widely, depending on the method of generation of bubbles, e.g. from the order of $10 \mu \mathrm{m}$ in electrolysis to the order of $1 \mathrm{~mm}$ in bubbling through a porous frit. The second purpose of the present paper is to make clear its effect in terms of the collection factor.

\section{Experimental}

The model emulsion was prepared from a heavy oil A with particles of about $8 \mu \mathrm{m}$ in arithmetic mean diameter, with a concentration of about $300 \mathrm{~g} \cdot \mathrm{m}^{-3}$. Details of the preparation and properties of the emulsion and analysis of the oil concentration were described in the previous paper ${ }^{6}$. The $\mathrm{pH}$ of liquid was adjusted to $\mathrm{pH} 4$ by addition of $\mathrm{HCl}$ solution so that double-layer repulsion was minimized as confirmed in the previous paper $^{\text {B) }}$ and so that the hydrodynamic effect may be observed in probably the most appropriate manner.

The separation of oil was carried out in two different systems of (1) bubbling and (2) electrolysis. Schematic diagrams are given in Figs. 1 (a) and (b), respectively. Each flotation column was a $0.5 \mathrm{~m}$-long acrylic cylinder of $65-\mathrm{mm}$ diameter. Liquid depth was kept constant at $32 \mathrm{~cm}$.

In a bubbling flotation, nitrogen rather than air was used for ready availability of a clean gas. Nitrogen was bubbled through porous glass frits of three different pore sizes and the bubble diameter varied over a range of $0.1-0.5 \mathrm{~cm}$ depending on frit pore size and gas flow rate. The residual oil concentration was analyzed after 30 minutes' bubbling.

In an electrolysis flotation, fine bubbles with an arithmetic mean diameter of $40 \mu \mathrm{m}$ were generated from a platinum electrode consisting of a spiralled wire and a squared plate as shown in Fig. 1 (b). Direct current was applied intermittently to avoid column flooding due to excessive bubbles encountered in continuous bubble generation. The circuit was closed for ten seconds for bubble generation and then opened for three minutes for flotation under mild flow conditions. After this cycle was repeated twelve times, the oil concentration was analyzed. While the circuit was closed, large nitrogen bubbles ( $\sim 5 \mathrm{~mm}$ in diameter) were introduced at the bottom with very low flow rate to facilitate the uniform dispersion of fine bubbles generated.

\section{Effect of Gas Flow Rate}

\section{1 Bubbling flotation}

Oil removal under various conditions is compared in terms of the oil concentration $C$ after 30 minutes' bubbling through frits. The particular value of 30 minutes' bubbling is selected because sufficient change in oil concentration was observed in this period, and satisfactory accuracy of analysis was obtained over the corresponding range of oil concentration. The results are plotted against the superficial gas velocity $U_{g}$ in Fig. 2 When the emulsion was left quiescent for 30 minutes, the oil concentration decreased to $80 \%$ of the initial value by spontaneous removal. The effect of frit size on residual concentration was unfortunately masked by much scattering in $C / C_{0}$. However, the effect of gas flow rate can be seen clearly. The residual concentration $C / C_{0}$ decreases slightly with gas rate $U_{g}$ at gas rate less than $0.05 \mathrm{~cm} \cdot \mathrm{s}^{-1}$ (zone 1) and decreases more rapidly at gas flow rate greater than $0.1 \mathrm{~cm} \cdot \mathrm{s}^{-1}$ (zone 2). At the transition between two zones the value of $C / C_{0}$ remains unchanged or even increases with increasing gas flow rate.

In some experimental runs, the variation of oil concentration with time was measured by sampling every 5 or 10 minutes. The average removal rates $\bar{r}$ per unit volume and unit time during the successive time intervals

$$
\bar{r}==\Delta C / \Delta t
$$

are plotted against time. The instantaneous removal 
rates $r$ were obtained by smoothing the $\bar{r}-t$ plot according to the method recommended by Churchill ${ }^{2}$.

The results are shown in Fig. 3 in the plot of $r / C$ vs. $C$. The value of $r / C$ is seen to be independent of $C$ in zone 1 while it is proportional to $C$ in zone 2 , resulting in the rate equations

$$
\begin{array}{ll}
r=k C & \text { in zone } 1 \\
r=k C^{2} & \text { in zone 2 }
\end{array}
$$

Oil separation by bubbling has been investigated by Kondo et al. ${ }^{3)}$, Saito et al. ${ }^{51}$, Angelldou et $a l^{11}{ }^{1)}$, and Takahashi et al. ${ }^{97}$ Their gas velocities were in the range of $0.1-15 \mathrm{~cm} \cdot \mathrm{s}^{-1}$, which are within the zone 2 of the present investigation. Angelldou et al. ${ }^{1)}$ reported a linear rate equation at $U_{g}=0.13 \mathrm{~cm} \cdot \mathrm{s}^{-1}$ while the recent result of Takahashi et al..$^{9}$ shows a rate equation of 2-3.5 order which increases with gas velocity in the range of $0.55-15 \mathrm{~cm} \cdot \mathrm{s}^{-1}$. Thus, the variation of the order of rate equation with gas velocity agrees with available reports although the critical gas velocity is different due to the difference of operating conditions such as bubble diameter. Takahashi et al. ${ }^{9 !}$ have proposed a two-parameter rate equation based on linear rate process with distributed rate constant. A power low rate equation of a single parameter is used for greater simplicity in the present work.

The first-order rate equation implies that a possible mechanism of separation in zone 1 is the collision of oil particles with rising bubbles, as in deep-bed filtration. The 2 nd-order rate equation in zone 2 implies that the collision of oil particles with each other is more important, as in flocculation sedimentation. When the emulsion was sampled during flotation, many agglomerates were observed through a microscope in the case of zone 2 but not in zone 1. This finding also supports the mechanism of oil particle collisions in zone 2 .

\section{2 Removal by mechanical agitation}

The effect of mechanical agitation was examined by mixing the emulsion with double-stage paddles having four blades of $39-\mathrm{mm}$ diameter and $19-\mathrm{mm}$ width for thirty minutes without bubbling. The result of the residual oil concentration is plotted in Fig. 2 against the power consumption per unit liquid volume $P_{v}$ which was estimated according to Nagata ${ }^{4}$. When the power for bubbling is taken to be

$$
P_{v}=\rho g U_{g}
$$

the corresponding power for bubbling can be scaled as shown in Fig. 2. The residual concentrations for bubbling flotation in zone 2 and for removal by agitation are shown to be the same at the same power consumption $P_{v}$. Thus, it can be concluded that the role of bubbling in zone 2 is to give suitable turbulence to the liquid and to give oil particles the kinetic energy

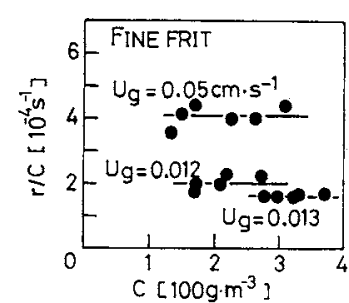

(a) ZONE 1

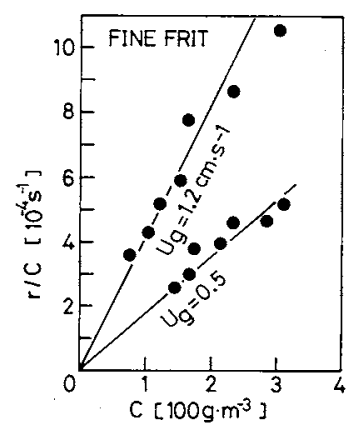

(b) ZONE 2

Fig. 3 Different dependence of removal rate on oil concentration between zone 1 and zone 2

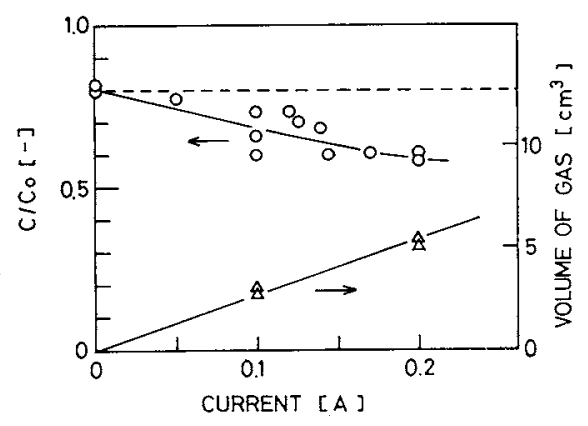

Fig. 4 Result of electrolysis flotation

required to collide and coagulate with each other.

\section{3 Electrolysis flotation}

The result for electrolysis flotation is shown in Fig. 4. The volume of bubbles evolved during one run was measured in a separate experiment and was found to be about $60 \%$ of the maximum attainable volume which can be estimated from Faraday's law, as shown in Fig. 4. The evolved gas volume at the highest current corresponds to the superficial gas velocity $U_{g}$ of $1.1 \times 10^{-3} \mathrm{~cm} \cdot \mathrm{s}^{-1}$ based on the net period of voltage application ( 2 minutes). Since this value of $U_{g}$ is much smaller than that in zone 1 of the bubbling flotation, the oil particles would be removed by the particle-bubble collision as in zone 1 . The residual oil concentration decreases here again with evolved gas volume or current.

\section{Effect of Bubble Diameter}

\section{1 General trend}

The electrolysis flotation, the bubbling flotation in zone 1 and the dissolved air flotation reported previ- 


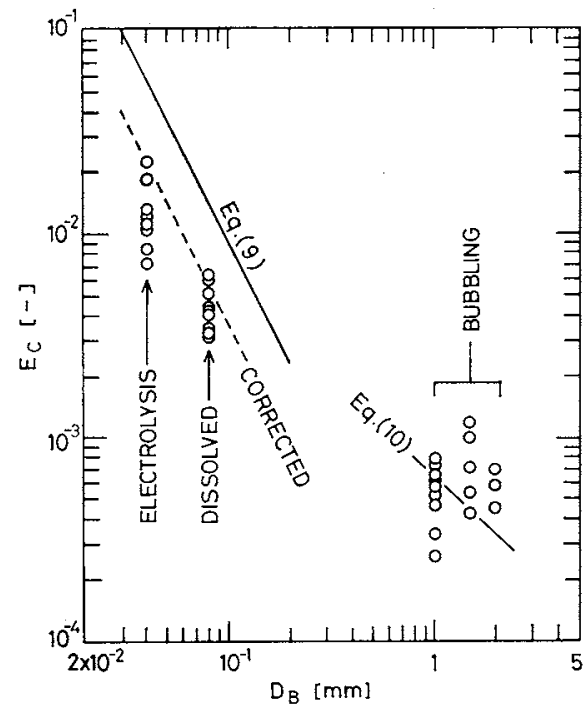

Fig. 5 Dependence of collection efficiency on bubble diameter

ously $^{6)}$ all proceed in particle-bubble collision and the approximate bubble diameters in these systems are summarized as follows:

Bubbling flotation (zone 1)

Coarse frit

Medium frit

$2 \mathrm{~mm}$

Fine frit

Dissolved flotation

$1.5 \mathrm{~mm}$

$1 \mathrm{~mm}$

Electrolysis flotation

$80 \mu \mathrm{m}$ (Ref. 6)

$40 \mu \mathrm{m}$

To see the general trend of the effect of bubble diameter, we considered three cases: (1) bubbling flotation at $U_{g}=10^{-2} \mathrm{~cm} \cdot \mathrm{s}^{-1}$; (2) dissolved flotation at a pressure of $2 \times 10^{5} \mathrm{~Pa}$ and (3) electrolysis flotation at a current of $0.2 \mathrm{~A}$. The residual oil concentrations are not much different among these cases, but the total bubble volumes consumed per unit liquid volume are quite different, i. e. $6 \times 10^{-1}, 3 \times 10^{-2}, 4 \times 10^{-3}$ for cases (1), (2) and (3), respectively. This fact indicates that the smaller bubble is the more effective in the flotation of oil particles.

\section{2 Collection efficiency $\boldsymbol{E}_{c}$}

The effect of bubble size may be twofold. One is the geometrical effect that small bubbles have a large projected area, facilitating collision with oil particles. The other is the hydrodynamic effect that the oil particle is more accessible to the smaller bubble. The latter effect can be evaluated in terms of the collection efficiency $E_{c}$ which is often used to characterize filtration performance. It is defined by the ratio of the number of oil particles collected by a bubble to that existing in the volume swept by the bubble.

The collection efficiency $E_{c}$ is related to the residual concentration at a particular point by a simple material balance, i. e.

$$
\left(\frac{C}{C_{0}}\right)_{10 \mathrm{cal}}=\exp \left\{-\frac{3}{2}-\frac{E_{c}}{D_{B}} v\right\}
$$

in which $v$ is the cumulative volume of bubbles which passes the column section of unit area. In bubbling flotation the value of $v$ is uniform with column height and equal to $U_{g} t$. Thus,

$$
-\frac{C}{C_{0}}=\exp \left\{-\frac{3}{2} \frac{E_{c} U_{g} t}{D_{B}}\right\}
$$

In electrolysis and dissolved flotations, bubbles are initially distributed uniformly and the quantity $v$ is proportional to the distance $x$ above the bottom according to $v=\alpha x$, where $\alpha$ is the volume of bubbles evolved per unit liquid volume. The observed concentration is the average along the height and the collection efficiency is given by the following equation:

$$
\frac{C}{C_{0}}=\frac{1}{h} \int_{0}^{h}\left(\frac{C}{C_{0}}\right)_{\text {local }} \mathrm{d} x=\frac{2}{3} \frac{D_{B}}{E_{\mathrm{c}} \alpha h}\left[1-\exp \left\{-\frac{3}{2} \frac{E_{c} \alpha h}{D_{B}}\right\}\right]
$$

The experimental values of $E_{c}$ were calculated from Eqs. (6) and (7) and are plotted against bubble diameter in Fig. 5. In the case of clectrolysis and bubbling flotations, the initial concentration $C_{0}$ was taken to be $80 \%$ of actual value, assuming that the spontaneous removal of $20 \%$ in 30 minutes took place independently of flotation.

\section{3 Comparison with theories}

When the oil particles colliding with the bubble are assumed to be all collected and removed, the collection efficiency $E_{c}$ may be predicted by a simple trajectory theory based on the interception:

$$
E_{c}=\text { maximum of }\left\{\frac{2 \psi}{U_{\infty}\left(D_{B} / 2\right)^{2}}\right\}_{y=D p / 2}
$$

where $\psi$ is the stream function of particle free flow around a bubble and $y$ is the distance from the bubble surface. Since the bubble behaves like a solid sphere, $E_{c}$ for creeping flow is given as ${ }^{8}$

$$
E_{c}=\frac{3}{2}\left(\frac{D_{P}}{D_{B}}\right)^{2}\left(1+\frac{2}{3} \frac{D_{P}}{D_{B}}\right) /\left(1+\frac{D_{P}}{D_{B}}\right)
$$

Similarly, $E_{c}$ for boundary layer flow is given as

$$
E_{c}=0.995\left(D_{P} / D_{B}\right)^{2} \sqrt{R e}
$$

of which the derivation is given in Appendix. Equations (9) and (10) are also shown in Fig. 5 for comparison with the experimental values.

The general trend of dependence of experimental collection efficiency $E_{c}$ on the bubble diameter $D_{B}$ can be found in Fig. 5 in spite of much scattering. With increasing diameter the factor $E_{c}$ decreases rapidly for small bubbles and much more gradually for large bubbles as the theories of Eqs. (9) and (10) predict, respectively. However, the measured $E_{c}$ for small bubbles falls below the $E_{c}$ predicted by Eq. (9). This difference may be in part explained by the modernized trajectory theory ${ }^{8}$, which takes into account many relevant forces as well as interception. The parameter $N_{a d}$ : 


$$
N_{a d}=\frac{Q\left(D_{B} / 2\right)^{2}}{9 \pi \mu\left(D_{P} / 2\right)^{4} U_{\infty}}
$$

is important in the present case with negligibly small gravity force and double-layer repulsion. The value of $N_{a d}$ with the most probable value of $Q=3 \times 10^{-3} \mathrm{~J}$ is $1.9 \times 10^{-3}$ and the theory of Spielman and Fitzpatrik ${ }^{8 ?}$ predicts the value of $E_{c}$ to be $40 \%$ of that given by Eq. (9), as shown by the broken line in Fig. 5 .

There are still some differences between the experimental and theoretical collection efficiency, probably because of partial collection of colliding oil particles, rather wide size distibution of bubbles and oil particles and so on. However, the available and proposed trajectory theories are useful since they predict the collection efficiency to within one order of magnitude at worst.

\section{Conclusions}

The following conclusions are derived for the flotation of oil particles in the absence of repulsion due to the electric double layer.

Firstly, the flotation rate in bubbling flotation through glass frit is proportional to the oil concentration to the first order at low gas velocity (zone 1, $\left.0.01 \leqq U_{g} \leqq 0.05 \mathrm{~cm} \cdot \mathrm{s}^{-1}\right)$ and to the second order (zone $2,0.1 \leqq U_{g} \leqq 3 \mathrm{~cm} \cdot \mathrm{s}^{-1}$ ). The predominant rate step may be oil particle-bubble collision in zone 1 and particle-particle collision in zone 2. Mechanical agitation and bubbling flotation in zone 2 give the same removal rate with the same power consumption. The role of bubbling in zone 2 is to give adequate turbulence to the liquid.

Secondly, with increasing bubble diameter, the collection efficiency for particle-bubble collision decreases rapidly ( $\simeq$ to the -2 power) for small bubbles and much more gradually for large bubbles. This trend coincides with the existing and proposed trajectory theories.

\section{Appendix: Derivation of Eq. (10)}

When the diameter of the oil particle is much smaller than the boundary layer thickness, the distribution can be approximated by the asymptotic one at the bubble surface, given by Schlichting $^{\text {?) }}$ as

$$
\frac{u}{U_{\infty}}=1.392 \sqrt{\frac{3}{2}}\left(1-0.3925 \theta^{2}+0.0421 \theta^{4}+\ldots\right) \sqrt{\overline{R e}} \frac{y}{\left(D_{B} / 2\right)}
$$

By definition of $\phi$ it follows that

$$
\begin{aligned}
& \frac{2 \phi}{U_{\infty}\left(D_{B} / 2\right)^{2}}=\frac{2 \sin \theta}{\left(D_{B} / 2\right)} \int_{0}^{D_{p} / 2} \frac{u}{U_{\infty}} \mathrm{d} y \\
& =\left[1.392 \sqrt{\frac{3}{2}} \cdot\left(1-0.3925 \theta^{2}+0.0421 \theta^{4}+\ldots\right) \sin \theta\right]\left(\frac{D_{P}}{D_{B}}\right) \sqrt{R e}
\end{aligned}
$$

The function in brackets has the maximum of 0.995 at $\theta=68^{\circ}$, and Eq. (10) is readily derived.

$$
\begin{aligned}
& \text { Nomenclature } \\
& C, C_{0} \quad=\text { residual and initial oil concentration, } \\
& \text { respectively } \\
& D_{B}, D_{P} \quad=\text { diameter of bubble and oil particle, } \\
& \text { respectively } \\
& E_{c} \quad=\text { collection efficiency } \\
& g \quad=\text { acceleration due to gravity } \quad\left[\mathrm{m} \cdot \mathrm{s}^{-2}\right] \\
& h \quad=\text { height of liquid } \\
& N_{a d} \quad=\text { parameter defined by Eq. (11) } \\
& P_{v} \quad=\text { power consumption per unit volume } \quad\left[\mathrm{W} \cdot \mathrm{m}^{-3}\right] \\
& Q \quad=\text { Hamaker constant [J] } \\
& \text { Re } \quad=\text { Reynolds number, } D_{B} U_{\infty} \rho / \mu \\
& r, \bar{r} \quad=\text { instantaneous and average rates of oil removal } \\
& \text { per unit volume and unit time, } \\
& \text { respectively }\left[\mathrm{g} \cdot \mathrm{m}^{-3} \cdot \mathrm{s}^{-1}\right] \\
& t \quad=\text { flotation time [s] } \\
& U_{g}, U_{\infty}, u=\text { superficial gas velocity, bubble rising } \\
& \text { velocity, local fluid velocity, } \\
& \text { respectively } \\
& v \quad=\text { cumulative volume of bubbles passing the } \\
& \text { column of unit area } \\
& =\text { distance from the bottom of flotation } \\
& \text { column } \\
& y=\text { distance from the bubble surface } \quad[\mathrm{m}] \\
& \alpha \quad=\text { bubble volume evolved per unit liquid } \\
& \text { volume } \\
& \theta=\text { angle from the front stagnation point } \\
& \mu, \rho=\text { viscosity and density of liquid, respectively } \\
& \phi \quad=\text { stream function }\left[\mathrm{m}^{3} \cdot \mathrm{s}^{-1}\right]
\end{aligned}
$$

\section{Literature Cited}

1) Angelldou, C., E. Keshavarz, M. J. Richardson and G. J. Jameson: Ind. Eng. Chem., Process Des. Dev., 16, 436 (1977).

2) Churchill, S. W.: "The Interpretation and Use of Rate Data-Rate Concept", Part III, McGraw-Hill, 1975.

3) Kondo, G., T. Hashimoto and S. Sano: Mizushori Gijutsu (Water Purification and Liquid Wastes Treatment), 13 (12), 41 (1972).

4) Nagata, S.: "Mixing: Principles and Applications", p. 24, Kodansha-John Wiley \& Sons, 1975.

5) Saito, T., K. Hagihara, Y. Ozasa and Y. Murakami: Mizushori Gijutsu (Water Purification and Liquid Wastes Treatment), 14, 823 (1973).

6) Sato, Y., Y. Murakami, T. Hirose, H. Yamamoto and Y. Uryu: J. Chem. Eng. Japan, 12, 454 (1979).

7) Schlichting, H.: "Boundary Layer Theory", p. 226 McGraw-Hill, 1968.

8) Spielman, L. A. and J. A. Fitzpatrik: J. Coll. Int. Sci., 42, 607 (1973).

9) Takahashi, T., T. Miyahara and Y. Nishizaki,: J. Chem. Eng. Japan, 12, 394 (1979).

(Presented at the Kagoshima Meeting of The Soc. of Chem., Engrs., Japan, at Kagoshima, 1977.) 